Voix et Images

volxetimages

\title{
L'Hexagone et la nationalisation de la poésie québécoise
}

\section{François Dumont}

Volume 15, numéro 1 (43), automne 1989

Jacques Poulin

URI : https://id.erudit.org/iderudit/200819ar

DOI : https://doi.org/10.7202/200819ar

Aller au sommaire du numéro

\section{Éditeur(s)}

Université du Québec à Montréal

\section{ISSN}

0318-9201 (imprimé)

1705-933X (numérique)

Découvrir la revue

\section{Citer cet article}

Dumont, F. (1989). L’Hexagone et la nationalisation de la poésie québécoise.

Voix et Images, 15(1), 93-101. https://doi.org/10.7202/200819ar d'utilisation que vous pouvez consulter en ligne.

https://apropos.erudit.org/fr/usagers/politique-dutilisation/ 


\title{
L'Hexagone et la nationalisation de la poésie québécoise
}

\author{
par François Dumont, université Laval
}

Le monde canadien est une branche de l'arbre français, une branche robuste et qui semble maintenant séparée du tronc original par une épaisse muraille: une branche quand même et qui fait honneur à l'arbre, à la vitalité de l'arbre.

Georges Duhamel

Si nous sommes l'arbre, jamais arbre ne s'est moins soucié de sa branche. $Q u^{\prime} i l$ s'en soucie aujourd'hui, rien de mieux, mais ce qu'il retrouve, après l'avoir si longtemps négligé, ce n'est plus une branche, c'est un arbre: un arbre de même espèce que lui, mais un autre arbre, qui est un arbre comme lui.

Étienne Gilson

Jusqu'à la fin du siècle dernier, on a dit des lettres de l'Amérique hispanique qu' elles étaient une branche du tronc espagnol. [...] En fait, c'est un autre arbre. Un arbre aux feuilles plus vertes et aux fruits plus amers. Dans ses bras nichent des oiseaux inconnus en Espagne.

Octavio Paz

La métaphore de l'arbre et de la branche revient souvent dans le discours sur l'autonomie des littératures coloniales. De cette façon, on renvoie celles-ci à leurs racines, à la littérature-mère, comme si la nationalisation n'était possible que par la rupture avec l'arbre originel. Au Québec, le discours «régionaliste» a ainsi longtemps voulu autonomiser la littérature canadienne-française en l'opposant à la littérature française (au «parisianisme»). Avec l'Hexagone, il semble que le projet de nationaliser la littérature - la poésie, dans un premier temps - ait suivi une autre voie, en focalisant non pas sur le rapport entre l'arbre et la branche, mais bien sur le chant des oiseaux aborigènes, sur les mots corbeaux de poème qui croassent ${ }^{1}$. Autrement dit, l'exemple de

1 Gaston Miron, «La braise et l'humus», l'Homme rapaillé, Montréal, P.U.M., 1970, p. 53. 
l'Hexagone montrerait que la nationalisation de la littérature n'est pas forcément un processus d'opposition; qu'elle peut être un processus de constitution, à partir de soi.

Le nom même d' «Hexagone» parait assez révélateur des visées de la maison d'édition, à sa naissance, en 1953. Jean-Louis Major rapporte en effet ces propos de Gaston Miron: Ce sera précisément le destin de l'Hexagone de réunir, autour d'une désignation aussi neutre, les valeurs et les aspirations de toute une génération littéraire. ${ }^{2}$ Le fait qu'on ait considéré «l'Hexagone», métonymie de la France, comme une désignation neutre, montre que le projet éditorial de la maison d'édition était bien éloigné de l'opposition à la littératuremère. Les poètes publiés par 1'Hexagone se référeront d'ailleurs fraternellement à de nombreux poètes français (René Char, Paul Éluard, Pierre-Jean Jouve, etc.). De son côté, la littérature canadienne-anglaise est carrément ignorée. Cela ne signifie pas pour autant que l'Hexagone n'ait pas voulu nationaliser la littérature québécoise. On reconnaît depuis longtemps cette volonté. Jean Royer, par exemple, a pu écrire que Gaston Miron, entouré d'Alain Horic et de quelques autres amis, a [...] provoqué, multiplié, diversifié - d cause et autour de l'Hexagone - une littérature nationale ${ }^{3}$. Le premier prospectus de l'Hexagone évoquait lui-même une littérature qui aurait une originalité et des caractéristiques qu' on lui reconnaît de plus en plus comme nationales ${ }^{4}$. Si ce projet remonte loin dans l'histoire du Québec, il est posé par l'Hexagone d'une façon nouvelle, selon une approche qui dépasse la traditionnelle opposition terroirisme/exotisme ${ }^{5}$, en exaltant non pas la différence, mais l'affirmation; en déplaçant la question de l'extérieur vers l'intérieur.

Je me limiterai, dans le cadre de cette étude, aux années 1953-1970, c'està-dire à la période qui va de la fondation de la maison d'édition à «la Nuit de la

2 Jean-Louis Major, «L'Hexagone: une aventure en poésie québécoise», Archives des lettres canadiennes, tome IV: la Poésie canadienne-française, Montréal, Fides, 1969, p. 177. Le nom d'«Hexagone» fut retenu parce que les fondateurs étaient six: Gaston Miron, Gilles Carle, Hélène Pilotte, Louis Portugais, Mathilde Ganzini et Olivier Marchand. À propos de l'histoire de la maison, on consultera, outre l'article de Major, le catalogue de l'exposition Hexagone 25, publié en 1979 par la Bibliothèque nationale.

3 Jean Royer, «En hommage à l'Hexagone», Estuaire, n® 9-10, décembre 1978, p. 4.

4 Cité par Gilles Marcotte dans «L'Hexagone et compagnie», Liberté, vol. XX, n० 6, novembre-décembre 1978, p. 19.

5 Il semble en effet que «terroiristes» et «exotistes» aient fondamentalement débattu du rapport à la France. La question aurait été de savoir à quelle France on voulait se rattacher (et, par le fait même, à quelle France on s'opposait). Camille Roy écrivait en 1907: Par [des] traditions conscientes ou quelquefois machinales, qui sont le fond de notre esprit, nous nous rattachons [...] étroitement à la France très chrétienne, à celle qui a précédé ou qui n'a pas fait la révolution (Essais sur la littérature canadienne, Québec, Librairie Garneau, 1907, p. 358). De son côté, Robert de Roquebrune affirmait, en 1918, dans le Nigog: Nous sommes nés de cette civilisation [française] et nous devons chercher à nous y rattacher. Que la France disparaisse un jour, et nous n'aurons plus de raison d'être (cité par Clément Moisan dans Comparaison et raison, Montréal, HMH, 1986, p. 13). 
poésie», récital qui précéda d'un an l'abandon par l'Hexagone de la formule de financement par souscription et la transformation de la Rencontre nationale des écrivains (qui fut d'abord une initiative de l'Hexagone) en Rencontre québécoise internationale des écrivains. Il semble que l'année 1970 - qui est aussi l'année de la publication, «hors Hexagone», de l'Homme rapaillé - constitue une année charnière pour la maison d'édition, et cela selon plusieurs points de vue: «la Nuit de la poésie» est la cristallisation d'un achèvement ${ }^{6}$; la structure de la maison change; une autre génération commence à s'affirmer en déléguant, déjà, dans une certaine mesure, aux poètes de l'Hexagone le rôle d' «arrièregarde»; à côté de l'Hexagone, de la Barre du jour et des Herbes rouges, s'élaborent d'autres lieux de poésie (les Écrits des Forges et les Éditions du Noroît naîtront en 1971); quant à l'aspect spécifiquement national du projet poétique de l'Hexagone, il est brutalement confronté aux prosaïques et incontournables forces de l'armée fédérale.

En m'attachant aux publications, aux activités et au discours de l'Hexagone, je tenterai de montrer comment le projet de nationaliser la littérature québécoise a été conçu et réalisé. Je m'attarderai d'abord sur l'«idéologie communautaire» des débuts, ensuite sur l'«espace national» tel qu'exprimé par la poésie de l'Hexagone et, finalement, sur la constitution d'une «référence» et d'une «durée» nationales.

Le tout premier recueil publié par l'Hexagone, Deux Sangs, est signé par deux auteurs: Olivier Marchand et Gaston Miron. Voilà déjà un signe de la manière de l'Hexagone et de ce qui la différencie de celle des «aînés». On a souvent dit combien, en poésie québécoise, les «aînés» étaient des solitaires. On s'imagine mal, par exemple, un recueil signé conjointement par Alain Grandbois et Anne Hébert, tant l'isolement était partie intégrante de leur condition et même de leur poétique. À l'Hexagone, la solitude est concrètement rompue par une nouvelle façon d'envisager la publication de la poésie. Deux Sangs, loin d'être une exception, inaugure un parti pris: la prééminence de la communauté sur l'individu. Des jours et des jours, les Clon̂tres de l'été, Ces anges de sang, affirme Gilles Marcotte, sont signés par l'Hexagone avant de l'être par Perrier, Pilon, Ouellette ${ }^{7}$. On pourrait aller jusqu'à dire que ces recueils, avant d'être signés par la maison d'édition et par les auteurs, auraient, par la formule de souscription, été signés par les lecteurs, par une communauté qui ressemble fort à un microcosme de la nation souhaitée: Nous croyons de cette façon réunir un assez grand nombre d'amis qui, au même titre que nous, seront les artisans de cette première publication. À cet extrait du premier prospectus de l'Hexagone, Marcotte ajoute: Joignant les indications du premier prospectus à celles du deuxième, nous pouvons dire que l'action de

6 ... sinon d'une mort. Laurent Mailhot et Pierre Nepveu affirment pour leur part: La récitation de «Speak white» par Michèle Lalonde aura probablement été le dernier mot de la poésie dite «nationales (la Poésie québécoise, Montréal, l'Hexagone, 1986, p. 31).

7 Gilles Marcotte, «L'Hexagone et compagnie», loc. cit., p. 15. 
l'Hexagone sera l'action du «milieu», que dans sa parole s'exprimera le «milieu». ${ }^{8} \mathrm{Du}$ milieu à la nation, il n'y a qu'un pas. Mais ce pas est difficile à franchir dans le contexte du début des années cinquante au Québec, en raison surtout de l'ambiguitté de l'idée de nationalisme à cette époque.

En 1953, au Québec, un nationalisme assez particulier est dans l'air. Cette année-là, Maurice Duplessis crée la Commission royale d'enquête sur les problèmes constitutionnels, dont le mandat implicite était de proclamer bien haut la légitimité des prétentions du gouvernement de la province de Québec à une large autonomie, tant financière que politique, au sein de la Confédération canadienne ${ }^{9}$. Il n'était pas possible, pour les poètes de l'Hexagone, de se reconnaître dans ce nationalisme, bien éloigné de la conception qu'ils se faisaient de la communauté, du «milieu». En effet, au début des années cinquante, et depuis plusieurs années déjà, le nationalisme québécois était lié au conservatisme, sinon à l'extrême droite. En raison, bien sûr, de l'ensemble des politiques de Maurice Duplessis. Mais aussi parce que depuis la fin de la Guerre, dans l'euphorie d'un nouvel esprit internationaliste, le nationalisme apparaissait tout à fait dépassé, associé aux tragédies dont on se relevait à peine ${ }^{10}$. L'opposition entre le «progrès social» et le nationalisme était ressentie par plusieurs intellectuels comme une contradiction insurmontable. C'est ainsi qu'en 1948, dans l'Action nationale, André Laurendeau écrivait:

Voilà que s'installe chez nous dans plusieurs secteurs une sorte de divorce entre ce que le langage courant appelle le social et le national. Ces mots sont ambigus. Et pourtant ils signifient dans la pratique des forces qui devraient être sours mais qui tendent à s'opposer. Notre tâche sera de les accorder ou plus exactement d' en faire la synthèse. ${ }^{11}$

Du côté de la littérature, le nationalisme était dans un dilemme du même ordre. D'une part, l'automatisme (mouvement qui, en poésie, précédait immédiatement l'Hexagone), à travers le Refus global, récusait fermement, dans un même souffle, la tuque et le goupillon. D'autre part, Robert Charbonneau, de la Nouvelle Relève, luttait pour l'autonomie nationale en matière d'édition, et ce désir «nationaliste» - canadien, celui-là - était perçu en France comme la volonté de publier des auteurs fascistes 12 .

8 Ibid.

9 Jean Hamelin et Jean-Paul Montminy, «La mutation de la société québécoise, 19391976. Temps, ruptures, continuités», Idéologies au Canada français, Québec, P.U.L., 1981, p. 45.

10 Louis Balthazar, Bilan du nationalisme au Québec, Montréal, l'Hexagone, 1986, p. 112.

11 Cité par Fernand Dumont dans le Sort de la culture, Montréal, l'Hexagone, 1987, p. 299.

12 Robert Dion («La France et nous après la Seconde Guerre mondiale. Analyse d'une crise», Volx \& images, vol. XIII, no 2, hiver 1988, p. 292-303) a montré que cet hypo-discours était fondé sur un hyper-discours d'ordre économique. Il n'empêche que l'hypo-discours traduisait d'une autre façon le malaise ressenti par Laurendeau. 
On comprend donc le malaise de Gaston Miron, à une époque où la relation entre nationalisme et socialisme était à l'aube d'un renversement:

$J$ 'avais répugné à militer plus tôt, en raison des origines de droite des premiers mouvements d'indépendance: mon option socialiste $m$ 'en éloignait. [...] C'est en poussant jusqu'à ses conséquences logiques mon socialisme, et par les études d'analyse sur notre société, que je concevais maintenant l'indépendance non plus seulement sur les plans de l'ontologie et du langage, mais sur le plan politique. [...] Mon engagement devait se traduire par des gestes de pair avec mon action en littérature et en édition. 13

C'est ainsi qu'un certain nombre de poètes, dont Miron et Michel van Schendel, substituent au lexème nationalisme le syntagme conscience nationale, voulant par là bien marquer la différence entre un point d'arrivée (la nation que nous avons) et un point de départ (la nation que nous aurons, et qui sera socialiste).

Une nouvelle relation se crée entre nationalisme et changement social. Mais il en est une autre qui fait problème pour plusieurs poètes de l'Hexagone: la relation entre le «social-national» et la poésie. La nature de ce lien sera l'objet de la première Rencontre nationale des écrivains, dont les actes seront publiés par l'Hexagone en 1958, sous le titre la Poésie et nous. Ce livre n'est pas un manifeste, comme le titre pourrait le laisser entendre. En effet, loin d'être l'expression d'une unanimité, il met au jour un conflit. Ce conflit demeure discrètement implicite jusqu'à l'intervention de Wilfrid Lemoine qui, sous l'intertitre "Avertissement», explique: On m'a demandé une communication sur «La Poésie et l' Homme» et on m' a indiqué comme sous-titre: la place de la poésie dans notre société et la fonction sociale du poète. Et il prend soin d'ajouter: L'expression «fonction sociale» du poète m' a fait voir rouge, au sens physiologique du terme. ${ }^{14}$ Lemoine n'est pas le seul poète à s'opposer au «fonctionnalisme» et à la présence du socialisme et du nationalisme dans le discours poétique. Les fortes dissensions à ce sujet aboutiront à une dissociation qui se fera de plus en plus nette entre le projet de nommer et celui de faire. Cette distinction se fera douloureusement: l'utopie du changement par le moyen de la poésie était forte, et trouvait des partisans tant du côté des poètes (de Parti pris, notamment) que du côté de la critique. Georges-André Vachon, par exemple, évoquait, en 1967, un Âge de la parole poétique, qui fait le monde ${ }^{15}$. En 1970, après «la Nuit de la poésie» et, surtout, les Mesures de guerre, la volonté de faire le pays au moyen de la poésie apparaîtra à plusieurs écrivains, dont Michel van Schendel, tenir d'une mystique essentialiste qui attribue aux mots les qualités de la chose et les pouvoirs de l'acte ${ }^{16}$. Ce diagnostic rejoint d'une

13 Gaston Miron, l'Homme rapaillé, op. cit., p. 119-120.

14 Wilfrid Lemoine, «La Poésie et l'Homme», la Poésie et nous, Montréal, l'Hexagone, 1958, p. 67.

15 Georges-André Vachon, «L’ère du silence et l'âge de la parole», Études françaises, vol. III, no 3, août 1967, p. 321. C'est Vachon qui souligne.

16 Michel van Schendel, «Mystique littéraire et poésie nationaliste», Socialisme québécois, no 20, avril-mai-juin 1970, p. 69. 
certaine façon celui que posait, dès 1958, Fernande Saint-Martin, qui disait lire les publications de l'Hexagone non comme de la poésie, mais comme de la prose qui s' ignore et qui n'ose pas aller au bout de ses possibilités ${ }^{17}$. Le même verdict fut récemment reconduit, à peu de choses près, par Jean Larose dans la Petite Noirceur ${ }^{18}$. Mais si le projet de faire par la poésie fut et est encore mis en cause, celui de nommer, lui, reste sans doute, au plan national, la réalisation la plus souvent créditée à l'Hexagone.

Nommer l'espace national ne fut cependant pas, tant s'en faut, l'exclusivité des poète de l'Hexagone. Plusieurs classiques de la «poésie du pays» des années soixante (l'Ode au Saint-Laurent de Gatien Lapointe ${ }^{19}$ ou Terre Québec de Paul Chamberland ${ }^{20}$, par exemple) ne furent pas publiés par l'Hexagone. De plus, de Jacques Cartier à Félix-Antoine Savard, en passant par les «terroiristes», nommer le pays fut une préoccupation constante dans l'écriture québécoise.

Dans ce contexte, les publications de l'Hexagone reflètent, sinon une exclusivité, du moins une tendance. En effet, on constate, par rapport à la thématique de la «fondation» dont a parlé Paul Chamberland ${ }^{21}$, que les publications de l'Hexagone témoignent, pour une part, du désir de nommer l'espace: un espace originel, d'avant la Conquête, et même d'avant la Découverte. Tout se passe comme si l'Hexagone proposait une poésie qui, dans l'imaginaire, tiendrait le rôle de l'épopée qui manque à la nation pour commencer. Par ailleurs, des recueils comme Pays sans parole ${ }^{22}$, Poèmes de l'Amérique étrangère ${ }^{23}$ ou Recours au pays ${ }^{24}$ témoignent à la fois d'une possibilité et d'un empêchement: possibilité retrouvée dans l'espace; empêchement provoqué par l'Histoire. Il ne s'agit pas, bien sûr, d'un réseau thématique constant et uniforme. Nombreux sont en effet les poètes de l'Hexagone qui ont peu à voir avec cette double thématique (Fernand Ouellette, par exemple, ou, jusqu'à un certain point, Paul-Marie Lapointe). Inversement, je l'ai déjà noté, d'autres maisons d'édition ont publié des poèmes «du pays» qui manifestaient, eux aussi, des tensions entre Origine et Histoire.

Il est tout de même assez remarquable que certains des recueils les plus imprégnés de désespérance à l'égard de la naissance du pays, certains des poèmes du plus «pur empêchement», ne seront pas publiés par l'Hexagone. À la "problématisation», l'Hexagone semble préférer un certain «adamisme» 25 qui,

17 Cité par Gilles Marcotte, loc. cit., p. 13.

18 Jean Larose, «Nationalisme et culture: les campagnes référendaires», la Petite Noirceur, Montréal, Boréal, 1987, p. 43-63.

19 Gatien Lapointe, Ode au Saint-Laurent précédé de J'appartiens à la terre, Montréal, Éditions du Jour, 1963.

20 Paul Chamberland, Terre Québec, Montréal, Déom, 1964.

21 «Fondation du territoire», Parti pris, vol. IV, nos 9-10-11-12, mai-août 1967, p. 1142.

22 Yves Préfontaine, Pays sans parole, Montréal, l'Hexagone, 1967.

23 Michel van Schendel, Poèmes de l'Amérique étrangère, Montréal, l'Hexagone, 1958.

24 Jean-Guy Pilon, Recours au pays, Montréal, l'Hexagone, 1961.

25 Le terme «adamisme» renvoie ici au mythe du nouvel Adam. Voir à ce sujet R.W.B. Lewis, The American Adam, Chicago, University of Chicago Press, 1971 et, pour 
sans béate sérénité, espère. La désespérance, l'empêchement, la patrie où l'on est très mal, se diront ailleurs: Mémoire ${ }^{26}$ paraît chez Déom puis chez Grasset; l'Homme rapaillé aux Presses de l'Université de Montréal; En désespoir de cause 27 chez Parti pris. Sans en faire un théorème, on peut donc constater que l'Hexagone, par rapport au destin national, privilégie le possible à la désillusion. Et ce possible vient avant tout de l'espace réinvesti par la parole. Si la parole poétique perdit, nous l'avons vu, peu à peu sa confiance de faire le futur, elle put tout de même influer d'une autre façon sur l'Histoire, en s'établissant comme passé: passé «littéraire», fondation tout de même.

Quand les «terroiristes» cherchaient leur spécificité, ils se tournaient vers la «nature canadienne», et la littérature nationale devenait un ensemble de réussites futures, fondées sur ce thème national: un avenir ${ }^{28}$. L'Hexagone, cherchant sa spécificité du même côté, la trouvait au passé: On trouve notamment chez Alain Grandbois ceci, qui est précieux pour l'avenir de la poésie canadienne: une expérience de l' espace [...]. Cet espace [...] commence à porter l'image au-delà de ses limites traditionnelles. ${ }^{29}$

Longtemps, en effet, la tradition littéraire fut perçue au Québec comme une limite:

[...] le mot littérature, quand il passe du domaine français au domaine québécois, change de sens. Là, il englobe un certain ensemble d'auvres incontestablement valables. Ici, il désigne la collection matérielle des auvres produites dans les limites d'un territoire national. [...] L'auvre ne m' est pas transmise. Elle n'a pas subi l'épreuve d' une tradition de lecture. [...] Or, tout indique que le Canada français est parvenu à [un] point optimum, depuis la dernière guerre. Le mécanisme profond de la création autochtone et de la lecture est déclenché; les premiers sédiments d' une tradition sont en train de se constituer, et nous savons, par exemple, ce que les poètes de l'Hexagone doivent à Grandbois. ${ }^{30}$

ce qui concerne l'expression de ce mythe dans la poésie québécoise, Maximilien Laroche, "L'image du nouveau-né et la figure du nouvel Adam dans la poésie québécoise», dans G. Dorion et M. Voisin (dir.), Littérature québécoise. Voix d'un peuple, voies d'une autonomie, Bruxelles, Editions de l'Université de Bruxelles, 1985, ainsi que mon article "Aspects du mythe de l'origine dans la poésie québécoise», dans Aux frontières du littéraire, Québec, CRELIQ, 1989.

26 Jacques Brault, Mémoire, Montréal, Déom, 1965; Paris, Grasset, 1968.

27 Pierre Perrault, En désespoir de cause. Poèmes de circonstances atténuantes, Montréal, Parti pris, 1971.

28 Voir à ce sujet l'article de Maurice Lemire: «L'autonomisation de la "littérature nationale" au XIX ${ }^{e}$ siècle», Études littéraires, vol. XX, nº 1, printemps-été 1987, p. 75-98.

29 Michel van Schendel, «Vues sur les tendances de la poésie canadienne-française», la Poésie et nous, op. cit., p. 18-19.

30 Georges-André Vachon, «Le domaine littéraire québécois en perspective cavalière», Histoire de la littérature française du Québec, tome I, Montréal, Beauchemin, 1967, p. $27,29,30$. 
Grandbois fut donc, particulièrement pour les poètes de l'Hexagone, la possibilité d'une tradition de lecture. Il ne fut pas tant un modèle qu'un point de départ, permettant l'inscription d'autres auvres à la suite d'une œuvre inaugurale. Ce point de départ permit aux poètes de l'Hexagone d'échapper au recommencement perpétuel de la littérature québécoise, qui ne connaissait, avant Grandbois, dans une certaine mesure, que l'exemplarité de l'échec. Fernand Ouellette évoquait, dès 1954, le mérite des îles de la nuit: Enraciner chez le lecteur un paysage d'émerveillement. Il poursuivait en affirmant: Par le chant noble d'Alain Grandbois qui m'avait précédé fraternel et beau, la montée pénible vers le sublime de la poésie m'était dès lors accessible. ${ }^{31}$

Grandbois, par sa référence, aurait «créé» l'Hexagone. D'une autre façon, l'Hexagone a aussi «créê» Grandbois. C'est en effet l'Hexagone qui, à partir de 1957 , édite sa poésie. De plus, la référence, si elle était disponible, fut tout de même revendiquée. Les poètes de l'Hexagone célèbrent Grandbois et, à travers lui, la pérennité de la poésie. Grandbois ne fut bien sûr pas le seul à permettre d'établir une durée puisque l'Hexagone publiait par exemple, en 1956, Rina Lasnier ${ }^{32}$; mais il fut de loin la plus importante référence de l'Hexagone. C'est d'ailleurs nul autre que Grandbois qui inaugure, en 1963, la collection «Rétrospectives» 33 .

Le nom de cette collection est quelque peu trompeur. En effet, si la création de «Rétrospectives» permet de regarder derrière soi, elle permet surtout la prospective. La prospective et l'unité. Car ce que bâtit la collection, c'est une assise: l'assurance pour les poètes que leur œuvre s'inscrit dans une continuité; l'assurance pour la poésie nationale que le projet collectif, tout en étant varié, est un seul et même projet. Ainsi, «Rétrospectives» accueillera, à la suite de Grandbois, des auteurs «maison» tels Jean-Guy Pilon ${ }^{34}$, Olivier Marchand ${ }^{35}$ et Fernand Ouellette ${ }^{36}$, mais aussi un grand nombre de poètes venus d' «ailleurs», notamment du surréalisme (Roland Giguère ${ }^{37}$ ou Gilles Hénault ${ }^{38}$ ). En fait, à travers «Rétrospectives», l'Hexagone, en réunissant par exemple Alphonse Piché ${ }^{39}$ et Nicole Brossard ${ }^{40}$, «aplanit» les différences, indexe les conflits sous la figure nationale ${ }^{41}$. Cette "réduction» est paradoxalement une ouverture: la

31 Fernand Ouellette, "Alain Grandbois et les jeunes poètes», Amérique française, vol. XII, no 6, décembre 1954, p. 473.

32 Rina Lasnier, Présence de l'absence, Montréal, l'Hexagone, 1956.

33 Alain Grandbois, Poèmes, Montréal, l'Hexagone, 1963.

34 Jean-Guy Pilon, Comme eau retenue, Montréal, l'Hexagone, 1968.

35 Olivier Marchand, Par détresse et tendresse, Montréal, l'Hexagone, 1971.

36 Fernand Ouellette, Poésie, Montréal, l'Hexagone, 1972.

37 Roland Giguère, l'Âge de la parole, Montréal, l'Hexagone, 1965.

38 Gilles Hénault, Signaux pour les voyants, Montréal, l'Hexagone, 1972.

39 Alphonse Piché, Poèmes, Montréal, 1'Hexagone, 1976.

40 Nicole Brossard, le Centre blanc, Montréal, l'Hexagone, 1978.

41 De la même façon, l'Hexagone «assimile» un certain nombre de maisons d'édition qui l'avaient immédiatement précédée (Cahiers de la File indienne, Erta, Editions de Malte, etc.) en rééditant leur catalogue et en publiant des auteurs qui avaient fait paraître chez ces éditeurs leurs premières œuvres. 
création d'un «texte national», où les écrivains peuvent se situer et, éventuellement, auquel ils peuvent aussi s'opposer.

De la poésie, l'assise du «texte national» se serait étendue à l'ensemble de la littérature québécoise. C'est du moins ce qu'affirmait récemment Victor-Lévy Beaulieu:

Relire toute notre poésie $m^{\prime}$ a révélé quelque chose de fabuleux: on peut y passer sa vie et $y$ trouver tout ce dont on a besoin, ce qui n'était pas le cas il y a cinquante ans. Et si tu ajoutes [...] les auteurs romanesques [...], tu t'aperçois que notre littérature est devenue assez costaude pour qu'on puisse vivre à l'intérieur d'elle sans avoir l'impression qu' on est en manque de quelque chose. 42

Et les écrivains qui voudront vivre «à l'intérieur» d'elle seront déportés - s'ils ne le font pas d'eux-mêmes - du côté des «influences» française et américaine ${ }^{43}$.

L'histoire de l'Hexagone est donc capitale dans le processus constitutif d'une littérature nationale au Québec. Une relation nouvelle entre littérature et «société nationale» s'est d'abord difficilement créée, relation ayant ceci de neuf que l'autonomisation de la littérature s'y réconciliait avec l'autonomisation de la nation: la littérature québécoise pouvait, enfin, à la fois être une forme et être fondée. L'espace était positivement nommé: une cosmogonie collective avait eu lieu, au moins dans l'imaginaire. L'intertexte national n'était plus seulement folklorique, il était aussi littéraire, et il appelait une suite...

42 Jean Royer, «Victor-Lévy Beaulieu. Inventer son propre bateau», le Devoir, 30 janvier 1988, p. D-1, D-8.

43 Voir par exemple Pierre Milot, «Tel Quel ou les conditions d'émergence des Herbes rouges», Voix \& images, vol. XIII, no 2, hiver 1988, p. 317-323 et Jean Larose, «Une modernité bien de chez nous. La Barre du jour», la Petite Noirceur, op. cit., p. 141-171. 